\title{
An Extended Fuzzy TODIM Approach for Multiple-Attribute Decision-Making with Dual-Connection Numbers
}

\author{
Irvanizam Irvanizam (D), ${ }^{1}$ Tarmizi Usman, ${ }^{2}$ Muhd Iqbal, ${ }^{1}$ Taufiq Iskandar, \\ and Marzuki Marzuki ${ }^{3}$ \\ ${ }^{1}$ Department of Informatics, Universitas Syiah Kuala, Banda Aceh 23111, Indonesia \\ ${ }^{2}$ Department of Mathematics, Universitas Syiah Kuala, Banda Aceh 23111, Indonesia \\ ${ }^{3}$ Department of Statistics, Universitas Syiah Kuala, Banda Aceh 23111, Indonesia \\ Correspondence should be addressed to Irvanizam Irvanizam; irvanizam.zamanhuri@unsyiah.ac.id
}

Received 17 December 2019; Revised 4 April 2020; Accepted 20 June 2020; Published 11 July 2020

Academic Editor: Katsuhiro Honda

Copyright (c) 2020 Irvanizam Irvanizam et al. This is an open access article distributed under the Creative Commons Attribution License, which permits unrestricted use, distribution, and reproduction in any medium, provided the original work is properly cited.

\begin{abstract}
The TODIM is a decision-making method that can examine the psychological behavior of decision-makers (DMs). However, the traditional TODIM method has still not been having the ability to overcome fuzzy information such as interval values and linguistic variables. This paper proposes an extended TODIM decision-making model for multiple-attribute decision-making (MADM) problems in a linguistic environment using dual-connection numbers (DCNs). The extended model uses linguistic variables in which the values of alternatives and criteria for both of them are formatted in the triangular fuzzy numbers (TFNs) to express the uncertain information. First, some definitions and basic operators of the TFNs and DCNs are introduced. Then, the way how to convert fuzzy information in forms of the TFNs into DCNs and the step how to transform each criterion weight value into a crisp value using the defuzzification of Minkowski are demonstrated. Furthermore, the traditional TODIM is improved to address MADM problems with DCNs, and detailed calculation steps in determining decisions are explained. Finally, an illustrative example which is a cadre selection problem is applied to demonstrate the conformity and validity of the extended TODIM method and to compare it with some other methods.
\end{abstract}

\section{Introduction}

Multiple-attribute decision-making (MADM) has now become a principal issue in decision science. This approach can determine the best and optimal alternatives from a finite set of alternatives. Hence, the approach has been widely applied in many territories such as transportation [1], management [2], energy [3], and industry [4-6]. However, along with the growing variety of case studies and the increasing involvement of decision-makers in decision-making, we still must consider ways how to express the fuzziness information in human perceptivity and give appropriate evaluation by optimal decision methods for MADM problems. So far, there are numerous approaches to evaluate this fuzziness such as fuzzy set (FS), intuitionistic fuzzy set (IFS), and set-pair analysis (SPA). By using fuzzy truth-membership (TM), Zadeh in [7] proposed
FSs to interpret fuzzy assessment information. Based on the concept of FS, Atanassov in [8] then introduced IFSs that contain TM and the artificiality membership (AM). However, the IFSs can only be used to incomplete information and they cannot address uncertain and certain information. Then, Zhao in [9] first introduced SPA to interpret the TM and AM; besides, it also analyzes the relationship between uncertain and certain information. The SPA utilizes the dual-connection number (DCN) to formulate TM under certain and uncertain circumstances. Moreover, it can also analyze mathematically the characteristics, interrelation, and relation of these two circumstances. Based on their advantages, DCNs have been widely applied in an augmentative number of sectors to support decision-makers (DMs) in making feasible and rational judgments. A very recently, Garg and Kumar in [10] have used the SPA with the DCN method under the IFS environment to 
resolve a real-life case in India. Then, Garg and Kumar in [11] have also utilized the concept of DCN in the SPA theory to integrate the TOPSIS method for measuring an exponential distance for selecting the best flyover construction company in India. Fu and Zhou in [12] presented the DCN based on the triangular fuzzy number (TFS) for choosing a cadre of an organization in China. Irvanizam et al. in [13] implemented an application that supports a local government to distribute some decent homes for impoverished families in Aceh.

For MADM problems, there exist two general methods to support DMs for selecting the optimal solution sets from various alternatives. One is a method using aggregation operators (AOs) to integrate alternative information to form an extensive value. This method tends to lose information during the integration process of the AOs. To handle this situation, there exists another method which is the use of classical decision-making approaches such as PROMETHEE [14], ELECTRE [15], and TOPSIS [16]. Those methods assume that, in the judgment process, DMs always give their perceptions in a rational way which does not suit all practical situations. For these cases, Gomes and Lima in [17] first introduced a decision-making method called TODIM for offering a solution to handle irrational judgments using the theory of perspective.

In cognitive psychology, the prospect theory, created by Kahneman and developed by Tversky, is a theory that delineates the way decision-makers (DMs) choose among probabilistic alternatives by involving DM behavior risk. The theory can help the DMs to determine decisions according to the potential value of gains and losses to have the appropriate outcome. In addition, this theory also synthesizes the aspects of declining sensitivity, reference dependency, and loss aversion [17]. In the aspect of reference dependence, the final outcomes are obtained through losses and gains based on a reference alternative. In the aspect of diminishing sensitivity, for gains, the DMs tend to be risk-averse, whereas, for losses, they like to be risk preference. Meanwhile, in the aspect of loss aversion, the DMs' attitudes to losses are much more sensitive to gains.

In recent years, many scientists have extensively conducted MADM research using the TODIM method. They generally attempted to extend this method by utilizing the theory of fuzzy set to solve the issue of uncertainty in the MADM problems. For instance, Yu et al. in [18] developed a new TODIM model for MADM in a group manner that has a large scale in solving multiple-granular unbalanced fuzzy information. The proposed model used unbalanced linguistic term sets to express uncertainty fuzzy information. After calculating gain and loss under the unbalance linguistic term sets, the classical TODIM approach was used to rank the alternatives. Wang et al. in [19] described a dubious linguistic model using TODIM for evaluating some emergency events. The model utilized the information in the fuzzy linguistic form to simulate the nonobjective information assigned by a decision-maker using the sets of the hesitant fuzzy linguistic model. Ren et al. in [20] proposed an extension of TODIM using the probabilistic dual hesitant under fuzzy environment to give a contribution for handling the uncertainty in decision-making problems. This extended TODIM method can define the aleatory and epistemic uncertainties in a single framework simultaneously. Besides, Ren et al. also compared the Pythagorean TODIM method with the Pythagorean fuzzy TOPSIS. The result showed that the extended method is more useful to cover MADM problems such as the Pythagorean fuzzy TOPSIS. Llamazares in [21] proposed a generalized TODIM method to overcome inconsistency of the paradoxes affecting the model weights. The proposed method introduced the properties of weight monotonicity and weight consistency to avoid these paradoxes. Wang and $\mathrm{Li}$ in [22] utilized a hybrid TODIM method to evaluate the environmental protection institution system (EPIS) in China by considering the hybrid information with real and grey numbers. Lourenzutti and Krohling in [23] introduced a novel approach to overcome data types of heterogeneous systematically. This method is called a modular explanation of the TODIM. As mentioned before, we can see that some fuzzy perspective approaches for their extension TODIM have adopted either the theories of traditional probability, uncertainty, or fuzzy sets. Meanwhile, the use of DCN in SPA can observe uncertainties and certainties through the aspects of identity-discrepancy-contrary (IDC). These aspects can depict the relationship between two objects which is not owned by other theories. It is nearly a kind of new uncertainty theory that is different from the fuzzy set and probability theories, but it can obtain those two theories under certain circumstances [24]. Therefore, it will be a great challenge to integrate the TODIM method and its preference information within DCN for handling a MADM problem.

Moreover, some publications describe applications of some decision-making methods in their real cases. Irvanizam et al. in [25] developed an application for selecting a smartphone using TODIM with TFNs. Each criterion value in forms of TFNs was changed into a crisp value using the mean value of the beta distribution. After determining gains and losses and using the classical TODIM, the application can successfully show the selected smartphone that is suitable for the user wanted. Ren et al. in [26] have implemented a smart application using the TODIM method with the Pythagorean fuzzy under the fuzzy environment. The application was applied to a MADM selection problem in determining a governor who manages a bank in Asia.

In many real decision-making processes, the assessment information and DM's judgment preferences may be in the form of linguistic variables and under fuzzy environment. In this kind of case, these two pieces of information can be expressed in terms of fuzzy sets such as TFNs, Pythagorean fuzzy numbers, and TrFNs. In this study, we utilize the characteristic of TFNs to convert the information-processing problem of TFNs to the information-processing problem of DCNs. This step guides us to obtain the DCN distance. Later on, because of the limited rationality of DMs and dissimilar preferences when addressing gain and loss, the TODIM method is applied to be a suitable instrument that can advise an appropriate selection and an optimum satisfaction for DMs. Additionally, DCNs are not only able to represent uncertain and certain information but they also can analyze characteristics, interrelation, and a relation of these two pieces of information. Based on the 
abovementioned analysis, it will be a good idea to improve TODIM with the DCN environment.

In this paper, we consider the limited rationality of DMs. An extended TODIM methodology with DCNs is introduced. As the contributions and innovations, we provide an extended TODIM methodology for the MADM with DCNs and explain the steps of the proposed approach in detail. Moreover, we show the conformity and validity of the proposed approach by comparison of two existing decisionmaking methods.

The leftover of this paper is sequentially described from Section 2 to Section 5. Section 2 describes preliminaries by reviewing some basic concepts of TFNs and DCNs. Later on, Section 2 also reviews some operations of TFNs and DCNs involving in the proposed extension TODIM procedure for MADM. In Section 3, we describe the steps of the proposed extension TODIM. Section 4 demonstrates the case study to show whether the DM's risk attitudes can give an influence on the decision-making results or not. We then compare the extended TODIM results with the results taken from $[12,27]$. Finally, Section 5 ends up in this paper with some conclusions.

\section{Preliminaries}

As the foundation of this study, we in this section will describe some basic definitions related to this research such as definitions of a TFN, trapezoidal fuzzy number ( $\operatorname{TrFN})$, linguistic variable, and dual-connection number. Additionally, we also introduce some basic mathematical operations that can be used to TFNs and DCNs.

Definition 1 (triangular fuzzy number/TFN). Let $\tilde{a}$ is a TFN denoted as $\tilde{a}=\left(a^{L}, a^{M}, a^{U}\right)$, then the membership function of $\tilde{a}$ is defined by

$$
\tilde{f}_{a}(x)= \begin{cases}\frac{x-a^{L}}{a^{M}-a^{L}}, & a^{L} \leq x \leq a^{M}, \\ \frac{a^{U}-x}{a^{U}-a^{M}}, & a^{M} \leq x \leq a^{U}, \\ 0, & \text { otherwise. }\end{cases}
$$

Definition 2 (trapezoidal fuzzy number/TrFN). Let $\widetilde{b}$ is trapezoidal fuzzy number denoted as $\widetilde{b}=\left(b^{L}, b^{M_{1}}, b^{M_{2}}, b^{U}\right)$, then the membership function of $\widetilde{b}$ is described by [28]

$$
\tilde{f}_{b}(x)= \begin{cases}\frac{x-b^{L}}{b^{M}-b^{L}}, & b^{L} \leq x \leq b^{M_{1}}, \\ 1, & b^{M_{1}} \leq x \leq b^{M_{2}}, \\ \frac{b^{U}-x}{b^{U}-b^{M_{2}},} & b^{M_{2}} \leq x \leq b^{U}, \\ 0, & \text { otherwise. }\end{cases}
$$

Definition 3. Assuming that $\tilde{b}_{1}=\left(b_{1}^{L}, b_{1}^{M}, b_{1}^{U}\right)$ and $\widetilde{b}_{2}=\left(b_{2}^{L}, b_{2}^{M}, b_{2}^{U}\right)$ are two TFNs, then the following expressions can be applied [12]:

$$
\begin{gathered}
\tilde{b}_{1}+\tilde{b}_{2}=\left(b_{1}^{L}+b_{2}^{L}, b_{1}^{M}+b_{2}^{M}, b_{1}^{U}+b_{2}^{U}\right), \\
\lambda \tilde{b}_{2}=\left(\lambda b_{2}^{L}, \lambda b_{2}^{M}, \lambda b_{2}^{U}\right), \quad \lambda>0 .
\end{gathered}
$$

Definition 4 (defuzzification of Minkowski). Let $\widetilde{a}=\left(a^{L}, a^{M}, a^{U}\right)$ is a TFN, then the defuzzification of Minkowski of $\widetilde{a}$ that has been presented in [29] is

$$
\chi=a^{L}+\frac{\left(a^{U}-a^{M}\right)}{4} .
$$

Definition 5 (inverse membership function). Let $f_{B}^{L}:\left[b^{L}, b^{M_{1}}\right] \longrightarrow[0, \omega]$ and $f_{B}^{R}:\left[b^{M_{2}}, b^{U}\right] \longrightarrow[0, \omega]$ are left and right membership functions of $\widetilde{b}$. Then, the inverse membership function of $f_{B}^{L}$ and $f_{B}^{R}$ are defined as follows [30]:

$$
\begin{aligned}
& g_{B}^{L}:[0, \omega] \longrightarrow[0,1], \\
& g_{B}^{R}:[0, \omega] \longrightarrow[0,1] .
\end{aligned}
$$

Definition 6. Let $\widetilde{b}=\left(b^{L}, b^{M_{1}}, b^{M_{2}}, b^{U}\right)$ be a $\operatorname{TrFN}$, then the centroid point of $\widetilde{b}$ is defined as follows [31]:

$$
C P(\tilde{b})=(\bar{x} p(\tilde{b}), \bar{y} p(\tilde{b}))
$$

where

$$
\left\{\begin{array}{l}
\bar{x} p(\tilde{b})=\frac{\int_{b^{L}}^{b^{M_{1}}} x f_{B}^{L}(x) \mathrm{d} x+\int_{b^{M_{1}}}^{b^{M_{2}}} x \mathrm{~d} x+\int_{b^{M_{2}}}^{b^{U}} x f_{B}^{R}(x) \mathrm{d} x}{\int_{b^{L}}^{b^{M_{1}}} f_{B}^{L}(x) \mathrm{d} x+\int_{b^{M_{1}}}^{b^{M_{2}}} \mathrm{~d} x+\int_{b^{M_{2}}}^{b^{U}} f_{B}^{R}(x) \mathrm{d} x} \\
\bar{y} p(\tilde{b})=\frac{\int_{0}^{1} y g_{B}^{L}(y) \mathrm{d} y+\int_{0}^{1} y g_{B}^{R}(y) \mathrm{d} y}{\int_{0}^{1} g_{B}^{L}(y) \mathrm{d} y+\int_{0}^{1} g_{B}^{R}(y) \mathrm{d} y} .
\end{array}\right.
$$

According to Definition 6, we can obtain the centroid point of $\widetilde{b}$ for the TrFN case as defined in equation (3):

$$
\left\{\begin{array}{l}
\bar{x} p(\tilde{b})=\frac{1}{3}\left[b^{L}+b^{M_{1}}+b^{M_{2}}+b^{U}-\frac{\left(b^{M_{2}} b^{U}\right)-\left(b^{M_{1}} b^{L}\right)}{\left(b^{M_{2}}+b^{U}\right)-\left(b^{M_{1}}+b^{L}\right)}\right], \\
\bar{y} p(\tilde{b})=\frac{1}{3}\left[1+\frac{\left(b^{M_{2}}\right)-\left(b^{M_{1}}\right)}{\left(b^{M_{2}}+b^{U}\right)-\left(b^{M_{1}}+b^{L}\right)}\right] .
\end{array}\right.
$$

As the form of TFN $\widetilde{a}=\left(a^{L}, a^{M}, a^{U}\right)$ is similar to the $\operatorname{TrFN}$, then the centroid point of $\widetilde{a}$ for the TFN is simply replaced by the variables of $b^{L}=a^{L}, b^{U}=a^{U}, b^{M_{1}}=a^{M}$, and $b^{M_{2}}=a^{M}$.

Definition 7 (the area of the centroid point). Let $\tilde{a}$ is a fuzzy number that can be either a TFN or $\operatorname{TrFN}$, then the area 
between the centroid (the centre of gravity) point denoted by $(x p(\tilde{a}), \bar{y} p(\tilde{a}))$ is defined [32]:

$$
S(\tilde{a})=\bar{x} p(\widetilde{a}) * \bar{y} p(\widetilde{a}) .
$$

Definition 8 (ordering fuzzy number). Let $\tilde{a}$ and $\tilde{b}$ are two arbitrary fuzzy numbers, then three following conditions are satisfied [32]:

$$
\begin{aligned}
& \text { If } S(\widetilde{a})>S(\widetilde{b}) \text {, then } \widetilde{a}>\widetilde{b} \\
& \text { If } S(\widetilde{a})<S(\widetilde{b}) \text {, then } \widetilde{a}<\widetilde{b} \\
& \text { If } S(\widetilde{a})=S(\widetilde{b}) \text {, then } \tilde{a}=\widetilde{b}
\end{aligned}
$$

A connection-number can be literally defined as a number generated from the connection-degree [24]. Generally, the connection-degree formula in the SPA is defined as

$$
u=\frac{S}{N}+\frac{F}{N} \beta+\frac{P}{N} \mathscr{C} .
$$

Equation (11) shows the variable $N$ is the total number of features, the variable $S$ is the number of identity-features, and the variable $P$ is the number of contrary-features. In addition, the variable $F$ is known as the number of features which are not either identity- or contrary-features and the value of $F$ can be reached from the formula $F=N-S-P$. Later on, $S / N, F / N$, and $P / N$ are also called identity-degree, discrepancy-degree, and contrary-degree, respectively. The variable $\beta$ is the coefficient of the discrepancy-degree, and it is a real value from -1 to 1 or $\beta \in[-1,1]$, whereas the variable $\mathscr{C}$ is the coefficient of the contrary-degree, and it is stated as -1 . When the values of $\beta$ and $\mathscr{C}$ are not to be considered, the variable $\beta$ and $\mathscr{C}$ can be pretended as the markers for the discrepancy-degree and the contrary-degree, respectively. Clearly, when we assume $p=S / N, q=F / N$, and $r=P / N$, then equation (11) can be shortly rewritten as

$$
u=p+q \beta+r \mathscr{C},
$$

where the variables $p, q$, and $r$ satisfy the equation $p+q+r=1$. Furthermore, when $r=0$, we obtain $u=p+q \beta$ which is called an identical different dual-connection number as known as the dual-connection number.

Definition 9 (dual-connection number). Let $u$ is a dualconnection number (DCN), then $u=p+q \beta$, where $p, q \in \mathbb{R}^{+}, \beta \in[-1,1]$, and $\mathbb{R}^{+}$is a positive real-number [33].

Definition 10. Assume that $u_{a}=p_{a}+q_{a} \beta$ and $u_{b}=p_{b}+q_{b} \beta$ are two dual-connection numbers, then the two basic mathematical operations can be applied [12]:

$$
\begin{aligned}
& u_{a}+u_{b}=\left(p_{a}+p_{b}\right)+\left(q_{a}+q_{b}\right) \beta, \\
& u_{a} \times u_{b}=\left(p_{a} p_{b}\right)+\left(p_{a} q_{b}+q_{a} q_{b}+p_{b} q_{a}\right) \beta .
\end{aligned}
$$

Definition 11. If $\tilde{a}=\left(a^{L}, a^{M}, a^{U}\right)$ is a TFN where $a^{L}<a^{M}<a^{U} \in \mathbb{R}^{+}$and $\mathbb{R}^{+}$is a positive real-number, then a $\mathrm{DCN}$ of $\widetilde{a}$ is [12]

$$
u_{a}=p_{a}+q_{a} \beta
$$

where

$$
\begin{aligned}
p_{a} & =a^{M}, \\
q_{a} & =\frac{\sqrt{\left(a^{M}-a^{L}\right)^{2}+\left(a^{M}-a^{U}\right)^{2}}}{2}, \\
\beta & \in[-1,1] .
\end{aligned}
$$

Definition 12. Assuming that $u_{\tilde{a}}=p_{a}+q_{a} \beta$ and $v_{b}=p_{b}+$ $q_{b} \beta$ are two DCNs, then the distance between these two DCNs is

$$
L\left(u_{\tilde{a}}, u_{b}\right)=\left|p_{a}-p_{b}\right|+\left|q_{a}-q_{b}\right| .
$$

A variable can be expressed in either the crisp or linguistic term. The crisp dataset is a dataset obtaining a real value such as grading point average (GPA), age, and Human Poverty Index (HPI), whereas the linguistic dataset is a dataset expression that can be one word or sentence presenting an information granular. All data used in this paper are linguistic datasets.

\section{The Proposed Extension TODIM Method}

Section 3 presents the proposed extension TODIM method with DCNs using the concept of conversion of triangular fuzzy numbers (TFNs). In order to apply the method of classical TODIM for MADM problems with DCNs, we attempt to extend the classical TODIM principle by combining the concepts of centroid (the centre of gravity) point of a TFN and identical different DCN. The proposed TODIM steps are described as follows:

Step 1: construct the decision-matrix $A=\left[\widetilde{a}_{i j}\right]_{m x n}\left(\widetilde{a}_{i j}=\right.$ $\left.\left(a_{i j}^{L}, a_{i j}^{M}, a_{i j}^{U}\right)\right)$ where $(i=1,2, \ldots, m),(j=1,2, \ldots, n)$, $m$ is the number of alternatives, and $n$ is the number of criteria in order to represent the fuzzy dataset.

Step 2: to reduce the influence of different lengths to the result of the matrix $A$, the matrix $A$ is transformed in normalization way to the matrix $Z=\left[\widetilde{z}_{i j}\right]_{m x n}\left(\widetilde{z}_{i j}=\right.$ $\left.\left(z_{i j}^{L}, z_{i j}^{M}, z_{i j}^{U}\right)\right)$ using

$$
\tilde{z}_{i j}= \begin{cases}\left(\frac{a_{i j}^{L}}{\sum_{i=1}^{m} a_{i j}^{U}}, \frac{a_{i j}^{M}}{\sum_{i=1}^{m} a_{i j}^{M}}, \frac{a_{i j}^{U}}{\sum_{i=1}^{m} a_{i j}^{L}}\right), & \text { for } j \in C_{\text {benefit }}, \\ \left(\frac{1 / a_{i j}^{U}}{\sum_{i=1}^{m}\left(1 / a_{i j}^{L}\right)}, \frac{1 / a_{i j}^{M}}{\sum_{i=1}^{m}\left(1 / a_{i j}^{M}\right)}, \frac{1 / a_{i j}^{L}}{\sum_{i=1}^{m}\left(1 / a_{i j}^{U}\right)}\right), & \text { for } j \in C_{\text {cost }} .\end{cases}
$$

Step 3: convert the normalized decision-matrix $Z=$ $\left[\widetilde{z}_{i j}\right]_{m x n}\left(\widetilde{z}_{i j}=\left(z_{i j}^{L}, z_{i j}^{M}, z_{i j}^{U}\right)\right)$ into the decision-matrix of identical different dual-connection number $U=\left[u_{i j}\right]_{m x n}\left(u_{i j}=c_{i j}+d_{i j} \beta\right)$ using 


$$
u_{i j}=\left\{\begin{array}{l}
c_{i j}=z_{i j}^{M}, \\
d_{i j}=\frac{\sqrt{\left(z_{i j}^{L}-z_{i j}^{M}\right)^{2}+\left(z_{i j}^{U}-z_{i j}^{M}\right)^{2}}}{2}, \quad \beta \in[-1,1] .
\end{array}\right.
$$

Step 4: receive the weight matrix $W=\left[\widetilde{w}_{j}\right]_{1 x n}\left(\widetilde{w}_{j}=\right.$ $\left.\left(w_{j}^{L}, w_{j}^{M}, w_{j}^{U}\right)\right)$ for alternative $A_{i}$ with respect to each criterion $C_{j}$ from the decision-maker.

Step 5: convert the TFN weight values into crisp values using defuzzification of Minowski or Definition 4 so that the criterion weight matrix $W$ is becoming $\widehat{W}=$ $\left[\omega_{j}\right]_{1 x n}$ where $\omega_{j}$ is a crisp value.

Step 6: normalize the TFN's weight so that the total of criterion weight is equal to 1 :

$$
\omega_{j}=\frac{\omega_{j}}{\sum_{j=1}^{n} \omega_{j}} .
$$

Step 7: compute the relative weight $\omega_{j r}$ of criterion $C_{j}$ to the criterion $C_{r}$ that can be expressed by

$$
\omega_{j r}=\frac{\omega_{j}}{\omega_{r}},
$$

where $\omega_{r}=\max \left\{\omega_{j} \mid(j=1,2,3, \ldots, n)\right\}$.

Step 8: calculate the distance of two DCNs $L\left(u_{i j}, u_{k j}\right)$ that can be adopted from equation (16):

$$
L\left(u_{i j}, u_{k j}\right)=\left|c_{i j}-c_{k j}\right|+\left|d_{i j}-d_{k j}\right| .
$$

Step 9: enumerate the dominance of each alternative $A_{i}$ over each alternative $A_{k}$ for each scheme of the criterion $C_{j}$ using equation (22). This can be performed as the classical TODIM methodology:

$$
\phi_{j}\left(A_{i}, A_{k}\right)= \begin{cases}\sqrt{\frac{\omega_{j r} L\left(u_{i j}, u_{k j}\right)}{\sum_{j=1}^{n} \omega_{j r}},} & \text { if }\left(S\left(\widetilde{a}_{i j}\right)>S\left(\widetilde{a}_{k j}\right)\right), \\ 0, & \text { if }\left(S\left(\widetilde{a}_{i j}\right)=S\left(\widetilde{a}_{k j}\right)\right), \\ -\frac{1}{\theta} \sqrt{\frac{\left(\sum_{j=1}^{n} \omega_{j r}\right) L\left(u_{i j}, u_{k j}\right)}{\omega_{j r}},} & \text { if }\left(S\left(\widetilde{a}_{i j}\right)<S\left(\widetilde{a}_{k j}\right)\right),\end{cases}
$$

where $\theta$ is a variable or parameter that indicates the losses attenuation factor. Hence, the matrix of dominance degree under the criterion $C_{j}$ is constructed as

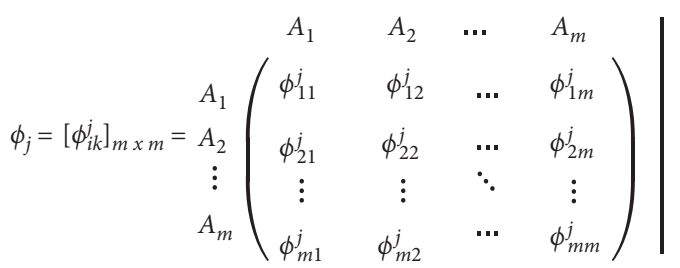

where $(k=1,2,3, \ldots, m ; j=1,2,3, \ldots, n)$.

Step 10: enumerate the degree of global dominance for each alternative $A_{i}$ over each alternative $A_{k}$ under the criterion $C_{j}$ by using

$$
\delta\left(A_{i}, A_{k}\right)=\sum_{j=1}^{n} \phi_{j}\left(A_{i}, A_{k}\right) .
$$

Step 11: normalize the matrix of global dominance degree in order to get the global value for each alternative $A_{i}$ by using

$\gamma\left(A_{i}\right)=\frac{\sum_{k=1}^{m} \delta\left(A_{i}, A_{k}\right)-\min _{i \in M}\left\{\sum_{k=1}^{m} \delta\left(A_{i}, A_{k}\right)\right\}}{\max _{i \in M}\left\{\sum_{k=1}^{m} \delta\left(A_{i}, A_{k}\right)\right\}-\min _{i \in M}\left\{\sum_{k=1}^{m} \delta\left(A_{i}, A_{k}\right)\right\}}$.

Obviously, the global value of each alternative $A_{i}$ will not be greater than 1 or less than 0 .

Step 12: select the best alternative in which the global value $\gamma\left(A_{i}\right)$ has the greater value through the final ranking order.

\section{Results and Discussion}

This section will show the results presented by using an illustrative example and compared to the obtained results of $[12,27]$. We also demonstrate a sensitivity analysis for conveying the conformity and validity of the extended fuzzy TODIM approach.

4.1. An Illustrative Example. This subsection will explain how the proposed fuzzy TODIM can be suitably applied to a cadre selection problem for an organization in China as obtained in [12]. The organization had to assess cadres based on six criteria (attributes): morality and ideology $\left(A T_{1}\right)$, working attitude $\left(A T_{2}\right)$, work custom $\left(A T_{3}\right)$, level of education and structure of knowledge $\left(A T_{4}\right)$, leadership $\left(A T_{5}\right)$, and ability of exploitation $\left(A T_{6}\right)$. Assume that there were 5 cadre candidates $\left(A_{1}, A_{2}, A_{3}, A_{4}\right.$, and $\left.A_{5}\right)$ who will be selected by the organization based on their highest scores. The criterion values of each attribute for each cadre candidate and the weight of each criterion are expressed in the form of TFNs. All criteria are previously defined as benefit criteria. 
TABle 1: The decision-making matrix.

\begin{tabular}{lcccccc}
\hline \multirow{2}{*}{ Alternative } & \multicolumn{5}{c}{ Criterion (attribute) } \\
& $A T_{1}$ & $A T_{2}$ & $A T_{3}$ & $A T_{4}$ & $A T_{5}$ & $A T_{6}$ \\
\hline$A_{1}$ & $(0.8,0.85,0.9)$ & $(0.9,0.92,0.95)$ & $(0.91,0.94,0.95)$ & $(0.93,0.96,0.99)$ & $(0.9,0.91,0.92)$ & $(0.95,0.97,0.99)$ \\
$A_{2}$ & $(0.9,0.95,1)$ & $(0.89,0.9,0.93)$ & $(0.9,0.92,0.95)$ & $(0.9,0.92,0.95)$ & $(0.94,0.97,0.98)$ & $(0.9,0.93,0.95)$ \\
$A_{3}$ & $(0.88,0.91,0.95)$ & $(0.84,0.86,0.9)$ & $(0.91,0.94,0.97)$ & $(0.91,0.94,0.96)$ & $(0.86,0.89,0.92)$ & $(0.91,0.92,0.94)$ \\
$A_{4}$ & $(0.85,0.87,0.9)$ & $(0.91,0.93,0.95)$ & $(0.85,0.99,0.9)$ & $(0.86,0.89,0.9)$ & $(0.87,0.9,0.94)$ & $(0.92,0.93,0.96)$ \\
$A_{5}$ & $(0.86,0.89,0.95)$ & $(0.9,0.92,0.95)$ & $(0.9,0.95,0.97)$ & $(0.91,0.93,0.95)$ & $(0.9,0.92,0.96)$ & $(0.85,0.87,0.9)$ \\
\hline
\end{tabular}

TABLE 2: The normalized decision-making matrix.

\begin{tabular}{lcccccc}
\hline \multirow{2}{*}{ Alternative } & \multicolumn{5}{c}{ Criterion (attribute) } \\
& $A T_{1}$ & $A T_{2}$ & $A T_{3}$ & $A T_{4}$ & $A T_{5}$ & $A T_{6}$ \\
\hline$A_{1}$ & $(0.17,0.19,0.21)$ & $(0.19,0.2,0.21)$ & $(0.19,0.2,0.21)$ & $(0.2,0.21,0.22)$ & $(0.19,0.2,0.21)$ & $(0.2,0.21,0.22)$ \\
$A_{2}$ & $(0.19,0.21,0.23)$ & $(0.19,0.2,0.21)$ & $(0.19,0.19,0.21)$ & $(0.19,0.2,0.21)$ & $(0.2,0.21,0.22)$ & $(0.19,0.2,0.21)$ \\
$A_{3}$ & $(0.19,0.2,0.22)$ & $(0.18,0.19,0.2)$ & $(0.19,0.2,0.22)$ & $(0.19,0.2,0.21)$ & $(0.18,0.19,0.21)$ & $(0.19,0.2,0.21)$ \\
$A_{4}$ & $(0.18,0.19,0.21)$ & $(0.19,0.21,0.21)$ & $(0.18,0.21,0.2)$ & $(0.18,0.19,0.2)$ & $(0.18,0.2,0.21)$ & $(0.19,0.2,0.21)$ \\
$A_{5}$ & $(0.18,0.2,0.22)$ & $(0.19,0.2,0.21)$ & $(0.19,0.2,0.22)$ & $(0.19,0.2,0.21)$ & $(0.19,0.2,0.21)$ & $(0.18,0.19,0.2)$ \\
\hline
\end{tabular}

TABle 3: The dual-connection number decision-making matrix.

\begin{tabular}{lcccccc}
\hline \multirow{2}{*}{ Alternative } & \multicolumn{5}{c}{ Criterion (attribute) $(u=p+q \beta)$} \\
& $A T_{1}$ & $A T_{2}$ & $A T_{3}$ & $A T_{4}$ & $A T_{5}$ \\
\hline$A_{1}$ & $0.19+0.014 \beta$ & $0.203+0.008 \beta$ & $0.198+0.008 \beta$ & $0.207+0.008 \beta$ & $0.198+0.005 \beta$ \\
$A_{2}$ & $0.213+0.015 \beta$ & $0.199+0.007 \beta$ & $0.194+0.009 \beta$ & $0.198+0.008 \beta$ & $0.211+0.007 \beta$ & $0.21+0.006 \beta$ \\
$A_{3}$ & $0.204+0.012 \beta$ & $0.19+0.008 \beta$ & $0.198+0.01 \beta$ & $0.203+0.008 \beta$ & $0.194+0.008 \beta$ & $0.199+0.007 \beta$ \\
$A_{4}$ & $0.195+0.01 \beta$ & $0.205+0.007 \beta$ & $0.209+0.015 \beta$ & $0.192+0.007 \beta$ & $0.196+0.009 \beta$ & $0.201+0.006 \beta$ \\
$A_{5}$ & $0.199+0.014 \beta$ & $0.203+0.008 \beta$ & $0.2+0.01 \beta$ & $0.2+0.007 \beta$ & $0.2+0.009 \beta$ & $0.188+0.007 \beta$ \\
\hline
\end{tabular}

TABLE 4: Weight, defuzzification, normalization, and the relative weight matrices.

\begin{tabular}{lcccccc}
\hline \multirow{2}{*}{ Weight } & \multicolumn{5}{c}{ Criterion (attribute) $(u=p+q \beta)$} \\
& $A T_{1}$ & $A T_{2}$ & $A T_{3}$ & $A T_{4}$ & $A T_{5}$ \\
\hline$W_{i}$ & $(0.19,0.21,0.23)$ & $(0.15,0.17,0.19)$ & $(0.1,0.13,0.15)$ & $(0.15,0.17,0.19)$ & $(0.1,0.13,0.15)$ & $(0.23,0.24,0.25)$ \\
Defuzzification & 0.195 & 0.155 & 0.105 & 0.155 & 0.105 & 0.2325 \\
Normalization & 0.206 & 0.164 & 0.111 & 0.164 & 0.111 & 0.245 \\
The relative weight $\omega_{j r}$ & 0.839 & 0.667 & 0.452 & 0.667 & 0.452 \\
\hline
\end{tabular}

4.2. Computation Steps and Results. In order to determine the most-right cadre from the selected candidates, we use the procedure of the proposed fuzzy TODIM approach introduced in Section 3. The results and the computation steps are sequentially explained in the following:

Step 1: construct a decision-making matrix A as presented in Table 1.

Step 2: determine a normalized decision-making matrix $Z$ using equation (18) as shown in Table 2.

Step 3: convert the matrix $Z$ into the DCN form as shown in Table 3.

Step 4: receive the weight matrix $W$ in form of TFNs as shown in Table 4.

Step 5: convert the matrix $W$ into crisp values using Definition 4.

Step 6: normalize the TFN's weight.

Step 7: compute the relative weight $\omega_{j r}$ of criterion $C_{j}$ to the criterion $C_{r}$.

Step 8: calculate the distance of two DCNs $L\left(u_{i j}, u_{k j}\right)$. Step 9: enumerate the dominance of each alternative $A_{i}$ over each alternative $A_{k}$ for each scheme of the criterion $C_{j}$. As an experiment, we in this study use $\theta=1$. It implies that there are contributions from the losses with the actual value to the global value [34]: 


$$
\left.\varnothing_{1}=\left(\begin{array}{ccccc}
0 & -0.335 & -0.273 & -0.200 & -0.211 \\
0.069 & 0 & 0.049 & 0.068 & 0.054 \\
0.056 & -0.237 & 0 & 0.047 & 0.036 \\
0.041 & -0.330 & -0.229 & 0 & -0.197 \\
0.043 & -0.264 & -0.173 & 0.041 & 0
\end{array}\right), \quad \gamma_{i}\right)=A_{3}\left(\begin{array}{c}
1.1666 \\
1.2044 \\
0.6091 \\
0 \\
A_{4}
\end{array}\right)
$$$$
\varnothing_{2}=\left(\begin{array}{ccccc}
0 & 0.029 & 0.048 & -0.134 & 0 \\
-0.178 & 0 & 0.041 & -0.202 & -0.178 \\
-0.291 & -0.250 & 0 & -0.320 & -0.291 \\
0.022 & 0.033 & 0.052 & 0 & 0.022 \\
0 & 0.029 & 0.048 & -0.134 & 0
\end{array}\right) \text {, }
$$$$
\varnothing_{3}=\left(\begin{array}{ccccc}
0 & 0.026 & -0.137 & 0.045 & -0.194 \\
-0.231 & 0 & -0.204 & 0.048 & -0.246 \\
0.015 & 0.023 & 0 & 0.042 & 0 \\
-0.403 & -0.431 & -0.379 & 0 & -0.354 \\
0.021 & 0.027 & 0 & 0.039 & 0
\end{array}\right) \text {, }
$$$$
\varnothing_{4}=\left(\begin{array}{ccccc}
0 & 0.039 & 0.029 & 0.053 & 0.036 \\
-0.240 & 0 & -0.163 & 0.035 & -0.135 \\
-0.178 & 0.027 & 0 & 0.044 & 0.022 \\
-0.321 & -0.213 & -0.267 & 0 & -0.231 \\
-0.223 & 0.022 & -0.134 & 0.038 & 0
\end{array}\right) \text {, }
$$$$
\varnothing_{5}=\left(\begin{array}{ccccc}
0 & -0.368 & 0.029 & 0.026 & -0.223 \\
0.041 & 0 & 0.045 & 0.044 & 0.037 \\
-0.258 & -0.409 & 0 & -0.166 & -0.249 \\
-0.234 & -0.394 & 0.018 & 0 & -0.211 \\
0.025 & -0.333 & 0.028 & 0.023 & 0
\end{array}\right) \text {, }
$$$$
\varnothing_{6}=\left(\begin{array}{ccccc}
0 & 0.048 & 0.054 & 0.046 & 0.074 \\
-0.195 & 0 & 0.030 & -0.053 & 0.057 \\
-0.219 & -0.124 & 0 & -0.112 & 0.055 \\
-0.188 & 0.013 & 0.027 & 0 & 0.057 \\
-0.300 & -0.232 & -0.223 & -0.234 & 0
\end{array}\right) .
$$

Step 10: enumerate the degree of global dominance $\delta\left(A_{i}, A_{k}\right)$ for each alternative $A_{i}$ over each alternative $A_{k}$ under the criterion $C_{j}$, and the result is shown in the following matrix:

$$
\delta=\left(\begin{array}{ccccc}
0 & -0.561 & -0.251 & -0.164 & -0.517 \\
-0.735 & 0 & -0.203 & -0.061 & -0.411 \\
-0.874 & -0.970 & 0 & -0.465 & -0.428 \\
-1.083 & -1.321 & -0.777 & 0 & -0.913 \\
-0.433 & -0.751 & -0.454 & -0.227 & 0
\end{array}\right) .
$$

Step 11: determine the normalization of the global dominance degree matrix $\gamma\left(A_{i}\right)$ :
Step 12: rank all alternatives based on the global value $\gamma\left(A_{i}\right)$ in descending order. The final result is $A_{2}>A_{1}>A_{5}>A_{3}>A_{4}$. The best or the right one is alternative $A_{2}$.

We then take the final result in terms of the ranking order to compare with other fuzzy MADM methods (setpair analysis [12] and ideal solution (IS) [27]). The comparison results show slight differences as shown in Figure 1.

As seen in Table 5, the results obtained from set-pair analysis and ideal solution methods are exactly the same. It is because the objectives of both methods are equal to build the ideal solutions and calculate the distance in various schemes. The method of ideal solution used the TFN to calculate the positive IS and negative IS, whereas the method of set-pair used the DCN to determine the absolute-positive and absolute-negative ideal DCNs. However, the result obtained from the proposed model is almost the same as the two others. The model showed that the alternatives $A_{5}$ and $A_{3}$ are being at the $3 \mathrm{rd}$ and 4 th positions, respectively, whereas the two others suggested the alternatives $A_{3}$ and $A_{4}$ are being at the third and fourth positions, respectively.

Even though the proposed method used the concept of the dual-connection number to convey the fuzzy information as presented by the set-pair method, the method compared the dual-connection number distance of all possible criteria pairs for each scheme to rank the alternatives. Hence, the obtained result in terms of ranking from the proposed method is slightly closer to the decisionmakers' perception than the two others. Once the organization in this case study had to select a single cadre, the three discussed decision methods can be applied and have suggested the same result.

4.3. Sensitivity Analysis. Sensitivity analysis is a simulation used in decision-making to analyze how the different parameter values give impacts on a specific decision under a certain range condition. In this case study, the simulation is used to predict whether there is an influence on the alternative ranking order or not when we modify the different values of losses $\theta$. According to Kahneman and Tversky in $[35,36]$, the parameter $\theta$ should be given a range from 1 to 2.5 .

In these experiments, we attempted to give different values of the parameter $\theta$ that ranged an interval from 1 to 2.5 and recalculated the ranking orders for each of the modified parameter value. As seen from Table 6, when we modify the value of $\theta$ from 1 to 2.5 , we see that the ranking order for each modified value of $\theta$ does not change. This indicates that the psychological behavior of DM in this case 


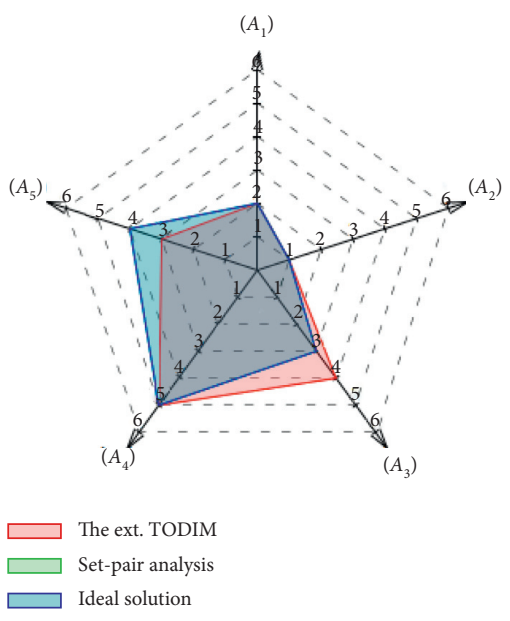

Figure 1: The comparison results.

TABLe 5: The comparison results.

\begin{tabular}{lc}
\hline Methods & The result \\
\hline The extended TODIM & $A_{2}>A_{1}>A_{5}>A_{3}>A_{4}$ \\
Set-pair analysis [12] & $A_{2}>A_{1}>A_{3}>A_{5}>A_{4}$ \\
Ideal solution [27] & $A_{2}>A_{1}>A_{3}>A_{5}>A_{4}$ \\
\hline
\end{tabular}

TABLE 6: The ranking-order results of alternatives for each different value of the parameter $\theta$.

\begin{tabular}{|c|c|c|c|c|c|c|}
\hline \multirow{2}{*}{$\begin{array}{l}\text { The } \\
\text { parameter } \\
\theta\end{array}$} & \multirow[b]{2}{*}{ The ranking order } & \multicolumn{5}{|c|}{$\gamma\left(A_{i}\right)$} \\
\hline & & $A_{1}$ & $A_{2}$ & $A_{3}$ & $A_{4}$ & $A_{5}$ \\
\hline$\theta=1$ & $A_{2}>$ & 1.666 & 1.2 & 0.6091 & 0 & 1 \\
\hline$\theta=$ & $A_{2}>A_{1}>A_{5}>A_{3}>A_{4}$ & 1.2060 & 1.2511 & 0.6134 & 0 & 1 \\
\hline$\theta=2$ & $A_{2}>A_{1}>A_{5}>A_{3}>A_{4}$ & 1.2437 & 1.2959 & 0.6175 & 0 & 1 \\
\hline$\theta=2.25$ & $A_{2}>A_{1}>A_{5}>A_{3}>A_{4}$ & 1.2620 & 1.3177 & 0.6185 & 0 & 1 \\
\hline$\theta=2.5$ & $A_{2}>A_{1}>A_{5}>A_{3}>A_{4}$ & 1.2799 & 1.3389 & 0.6215 & 0 & 1 \\
\hline
\end{tabular}

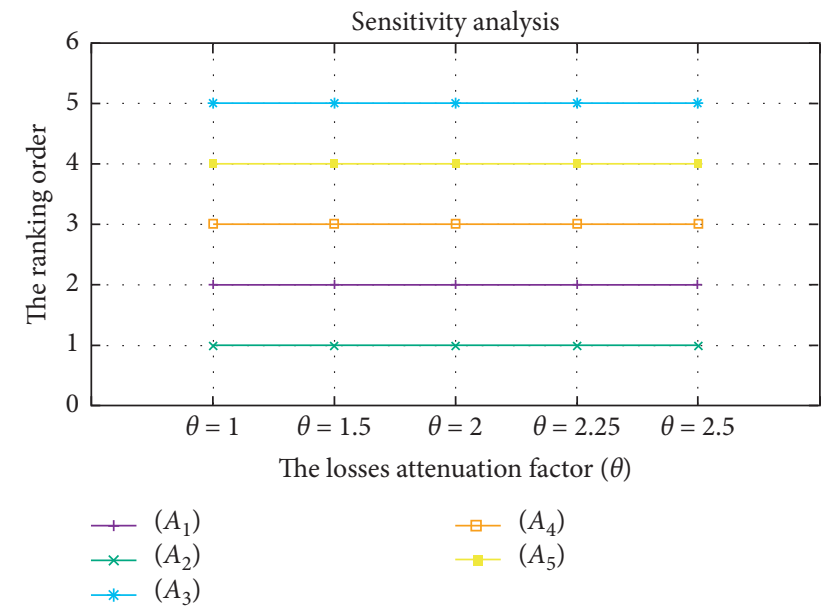

FIgURE 2: The sensitivity analysis. study does not affect the selection process in the decisionmaking. This analysis can be seen in Figure 2 .

\section{Conclusions and Future Studies}

This research proposes an extended TODIM that based on TFNs for handling the problems under a multiattribute fuzzy decision-making environment. It shows in a detailed manner how to utilize the concepts of DCNs in expressing uncertain information and select the appropriate alternatives using the TODIM in considering the DM judgments on the given alternatives. In addition, this also demonstrates how the alternatives' values in form of TFNs are transformed into DCNs and the weight values in form of TFNs are converted into crisp values through a defuzzification process using the defuzzification of Minkowski. By adopting the classical TODIM methodology, the matrix of dominance degree can be constructed, and the ranking order based on the global value of each alternative can be performed. In the end, we resolve a cadre selection problem for an organization in China reviewed from [12] to prove the conformity and validity of the extended TODIM method. In comparison with set-pair analysis and ideal solution methods, even though the extended TODIM can give a chance to the DM with her or his psychological behavior to affect the fuzzy information, it returned almost the same result in terms of the ranking order with the two others.

In the future study, it will be a worthy idea to combining the TODIM method with some other traditional decision methods, such as EDAS, AHP, PROMETHEE, and ELECTRE, and further consider other computation approaches of connection-degree such as gradual analysis, enumeration, hierarchical analysis, and statistical approaches to generate connection numbers. In addition, this research could be continued by reextending the calculation procedure with anticipation that a new method can be applied appropriately for other similar personal selection cases, such as manager selection, scholarship recipient selection, school principal selection, and others.

\section{Data Availability}

The data we used in this paper are taken from [12].

\section{Conflicts of Interest}

The authors declare that they have no conflicts of interest.

\section{Acknowledgments}

The authors thank the Department of Informatics, Universitas Syiah Kuala, for material support on this research.

\section{References}

[1] Y. Jingning, "The urban road soft foundation treatment scheme evaluation model based on the multiple attribute decision making theory," in Proceedings 2017 International Conference on Smart Grid and Electrical Automation (ICSGEA), pp. 576-579, Changsha, China, May 2017. 
[2] S. Sakhuja, V. Jain, and F. Dweiri, "Application of an integrated MCDM approach in selecting outsourcing strategies in hotel industry," International Journal of Logistics Systems and Management, vol. 20, no. 3, pp. 304-324, 2015.

[3] H.-C. Liu, M. Yang, M. Zhou, and G. Tian, "An integrated multi-criteria decision making approach to location planning of electric vehicle charging stations," IEEE Transactions on Intelligent Transportation Systems, vol. 20, no. 1, pp. 362-373, 2019.

[4] K. Chiao, "Interval type 2 fuzzy sets multiple criteria decision making based on quantifier guided aggregation ordered weighted averaging operator," in Proceedings of the 2017 Joint 17th World Congress of International Fuzzy Systems Association and 9th International Conference on Soft Computing and Intelligent Systems (IFSA-SCIS), pp. 1-6, Otsu, Japan, June 2017.

[5] K. Chiao, "Multiple criteria decision making using parametric graded mean integration representation with preference index on ranking interval type 2 fuzzy sets," in Proceedings of the 2016 International Conference on Fuzzy Theory and its Applications (iFuzzy), pp. 1-6, Taichung, Taiwan, November 2016.

[6] C. Kasemset and C. Boonmee, "An integration method of MFCA, dynamic programming, and multiple criteria decision making in operations improvement: a case study," in Proceedings of the 2017 IEEE International Conference on Industrial Engineering and Engineering Management (IEEM), pp. 745-749, Singapore, December 2017.

[7] L. A. Zadeh, "Fuzzy sets," Information and Control, vol. 8, no. 3, pp. 338-353, 1965.

[8] K. T. Atanassov, "Intuitionistic fuzzy sets," Fuzzy Sets and Systems, vol. 20, no. 1, pp. 87-96, 1986.

[9] K.-G. Zhao, "Set pair and set pair analysis-a new concept and systematic analysis method," in Proceedings of National System Theory and Regional Analysis Conference, pp. 87-91, Baotou, China, 1989.

[10] H. Garg and K. Kumar, "Power geometric aggregation operators based on connection number of set pair analysis under intuitionistic fuzzy environment," Arabian Journal for Sciences and Engineering, vol. 45, no. 3, pp. 2049-2063, 2019.

[11] H. Garg and K. Kumar, "A novel exponential distance and its based TOPSIS method for interval-valued intuitionistic fuzzy sets using connection number of SPA theory," Artificial Intelligence Review, vol. 1, pp. 1-20, 2018.

[12] S. Fu and H. Zhou, "Triangular fuzzy number multi-attribute decision-making method based on set-pair analysis," Journal of Software Engineering, vol. 11, no. 1, pp. 116-122, 2017.

[13] I. Irvanizam, I. Syahrini, R. P. F. Afidh, M. R. Andika, and H. Sofyan, "Applying fuzzy multiple-attribute decision making based on set-pair analysis with triangular fuzzy number for decent homes distribution problem," in Proceedings of the 2018 6th International Conference on Cyber and IT Service Management (CITSM), vol. 1, pp. 1-7, Parapat, Indonesia, August 2018.

[14] B. Mareschal, J. P. Brans, and P. Vincke, PROMETHEE: A New Family of Outranking Methods in Multicriteria Analysis, ULB, Universite Libre de Bruxelles, Brussels, Belgium, 1984.

[15] B. Roy and P. Bertier, La méthode ELECTRE II: une méthode de classement en prédence de critères multiples, Direction Scientifique, Note de Travail, paris, France, 1971.

[16] C. L. Hwang and K. S. Yoon, Multiple Attribute Decision Methods and Applications, Springer, Berlin, Germany, 1981.

[17] L. Gomes and M. Lima, "TODIM: basics and application to multicriteria ranking of projects with environmental impacts," Foundations of Computing and Decision Sciences, vol. 16, pp. 113-127, 1992.

[18] W. Yu, Z. Zhang, and Q. Zhong, "A TODIM-based approach to large-scale group decision making with multi-granular unbalanced linguistic information," in Proceedings of the 2017 IEEE International Conference on Fuzzy Systems (FUZZIEEE), pp. 1-6, Naples, Italy, July 2017.

[19] L. Wang, Y. Wang, R. M. Rodríguez, and L. Martinez, “A hesitant fuzzy linguistic model for emergency decision making based on fuzzy TODIM method," in Proceedings of the 2017 IEEE International Conference on Fuzzy Systems (FUZZIEEE), pp. 1-6, Naples, Italy, July 2017.

[20] Z. L. Ren, Z. S. Xu, and H. Wang, "An extended TODIM method under probabilistic dual hesitant fuzzy information and its application on enterprise strategic assessment," in Proceedings of the 2017 IEEE International Conference on Industrial Engineering and Engineering Management (IEEM), pp. 1464-1468, Singapore, December 2017.

[21] B. Llamazares, "An analysis of the generalized TODIM method," European Journal of Operational Research, vol. 269, no. 3, pp. 1041-1049, 2018.

[22] F. Wang and H. Li, "Novel method for hybrid multiple attribute decision making based on TODIM method," Journal of Systems Engineering and Electronics, vol. 26, no. 5, pp. 1023-1031, 2015.

[23] R. Lourenzutti and R. A. Krohling, "TODIM based method to process heterogeneous information," Procedia Computer Science, vol. 55, pp. 318-327, 2015.

[24] Y. L. Jiang, C. F. Xu, Y. Yao, and K. Zhau, "System information in set pair analysis and its applications," in Proceedings of the third International Conference on Machine Learning and Cybernetics, pp. 1717-1722, Shanghai, China, August 2004.

[25] I. Irvanizam, M. Marzuki, I. Patria, and R. Abubakar, "An application for smartphone preference using TODIM decision making method," in Proceedings of the 2018 International Conference on Electrical Engineering and Informatics (ICELTICs), pp. 122-126, Banda Aceh, Indonesia, September 2018.

[26] P. Ren, Z. Xu, and X. Gou, "Pythagorean fuzzy TODIM approach to multi-criteria decision making," Applied Soft Computing, vol. 42, pp. 246-259, 2016.

[27] Y. J. Xu and Q. L. Da, "Method for triangular fuzzy number multiple attribute decision making based on ideal solution," Systems Engineering and Electronics, vol. 29, no. 9, pp. 1469-1471, 2007.

[28] J. Q. Wang and L. Gong, "Interval probability stochastic: multi-criteria decision-making approach based on set pair analysis," Control Decision, vol. 24, pp. 1877-1880, 2007.

[29] M. Ghasemi, N. M. Yaghoubi, and A. N. Zehi, "Identifying and ranking the strong points and weaknesses of entrepreneurship development in the mine sector of Sistan and Baluchestan," International Journal of Humanities and Cultural Studies, vol. 1, pp. 903-919, 2016.

[30] L. Abdullah and N. N. A. Fauzee, "Ranking fuzzy numbers and its application to products attributes preferences," International Journal on Advanced Science, Engineering and Information Technology, vol. 1, no. 1, pp. 21-25, 2011.

[31] C.-H. Cheng, "A new approach for ranking fuzzy numbers by distance method," Fuzzy Sets and Systems, vol. 95, no. 3, pp. 307-317, 1998.

[32] T. C. Chu and C. T. Tsao, "Ranking fuzzy numbers with an area between the centroid point and original point," Computers \& Mathematics with Application, vol. 43, no. 1-2, pp. 111-117, 2002. 
[33] L. Hu, H. Yuan, and Q. Wu, "Multiple attribute decisionmaking of triangular fuzzy number based on set pair analysis," Journal Wuhan University Technology, vol. 37, pp. 108-111, 2015.

[34] X. Zhang and Z. Fan, "A method for linguistic multiple attribute decision making based on TODIM," in Proceedings of the 2011 International Conference on Management and Service Science, pp. 1-4, Wuhan, China, August 2011.

[35] A. Tversky and D. Kahneman, "Advances in prospect theory: cumulative representation of uncertainty," Journal of Risk and Uncertainty, vol. 5, no. 4, pp. 297-323, 1992.

[36] A. Mardani, A. Jusoh, K. Md Nor, Z. Khalifah, N. Zakwan, and A. Valipour, "Multiple criteria decision-making techniques and their applications-a review of the literature from 2000 to 2014," Economic Research-Ekonomska Istraživanja, vol. 28, no. 1, pp. 516-571, 2015. 